\title{
Surgical treatment of the sleep apnea Syndrome
}

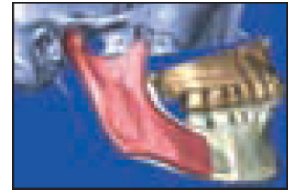

\author{
Boris PÉTELLE, Bernard FLEURY, \\ Julia COHEN-LÉVY
}

\section{ABSTRACT}

Treatment of severe cases of adult obstructive sleep apnea Syndrome is a life-long process. Consisting of every-night wear of a continuous pressure ventilation mask or the use of an oral appliance, side effects can appear a constraint and lead to progressive abandon of treatment.

Maxillo-mandibular advancement surgery allows a global and stable treatment of pharyngeal collapsus. Patient selection for this surgical procedure is difficult; a multidisciplinary team approach is mandatory, gathering a sleep physician, an orthodontist and surgeon. Occlusal analysis is associated with a cephalometric study, to look for skeletal abnormalities that could favour obstruction of the pharynx; the chosen protocol then aims at simultaneous treatment of apneic events and orthognathic abnormalities.

\section{KEYWORDS}

Obstructive sleep apnea syndrome

Maxillo-facial surgery

Facial aesthetic. 


\section{1 - INTRODUCTION}

Continuous positive airway pressure to assist nocturnal breathing is the reference treatment for obstructive sleep apnea syndrome (OSAS). It prevents closure of the pharynx during breathing by creating a pneumatic splint. Such a technique, however, cannot be considered a definitive solution for some patients because of the cumbersome nature of this device, which must be used for a lifetime. The alternative therapy currently being widely recommended is a mandibular advancement orthesis whose efficacy derives from the mechanical force the propulsion of the tongue exerts on the muscular mass of the tongue. This procedure enjoys a higher compliance rate than the positive airway pressure scheme and evaluations of it in the literature report that for 60 to $80 \%$ of patients it is deemed to work satisfactorily. But long term wearing of this appliance frequently leads to dento-alveolar movements that cause unwanted changes in occlusion. In order to avoid these undesirable side effects a surgical solution can be proposed.

Isolated soft palate surgery or more extensive removal of palatal and tonsillar soft tissue comprising a uvular-

\section{2 - PRE-OPERATIVE PROTOCOL}

The decision of which patients to select from the number of potential candidates for maxillomandibular surgery is a delicate one $2,8,12,19,20,25,26$. While the objective of this intervention is to correct nocturnal respiratory difficulty, this goal cannot be achieved palatal-pharyngoplasty (UPPP) can be considered for patients with light to moderate OSAS (apnea hypopnea per hour $\mathrm{IAH}<30 / \mathrm{h})$ with no cardiovascular comorbidity, no obesity (IMC > 30), or no evident velo-tonsillar or retro basilingual soft tissue blockage.

For patients afflicted with severe OSAS (IAH > 30/h) or burdened with blockage basically consisting of a poorly situated tongue, the most consistently effective surgical solution is maxillomandibular advancement osteotomy carried out with the same procedures used in orthognathic surgery. The objective of this intervention is to accomplish what an oral appliance would, displace the position of the base of the tongue anteriorly. This solution, initiated in the United States at Stanford University, achieves a definitive elimination of the apnea disorders in $90 \%$ of the cases ${ }^{20}$. In order to obtain these surgical results, surgeons must advance the mandible from 10 to $12 \mathrm{~mm}$. In contrast to soft palate surgery, these results are stable over the long term except for the lessened breathing capacity that can occur when patients gain a considerable amount of weight ${ }^{5,12}$.

without changing the patient's profile in ways that vary according to the facial equilibrium of the candidate. To make this decision, a multi-disciplinary team of sleep specialist, orthodontist, and surgeon must all contribute to evaluating the constraints and risks that this 
project engenders. An occlusal analysis, reinforced by a cephalometric assessment, is completed to uncover any possible skeletal anomalies that might be causing pathological obstruction.

The results of surgical mandibular advancement approximate what occurs when patients wear an oral appliance, a consideration that plays a useful role for some in the selection process $^{11}$. A pre-treatment simulation of the facial transformation that surgery would accomplish would be useful because for some patients the change would be considered advantageous but for others it would be deleterious $^{24}$ (see case 1, illustrated by figures 1 to 3 ).
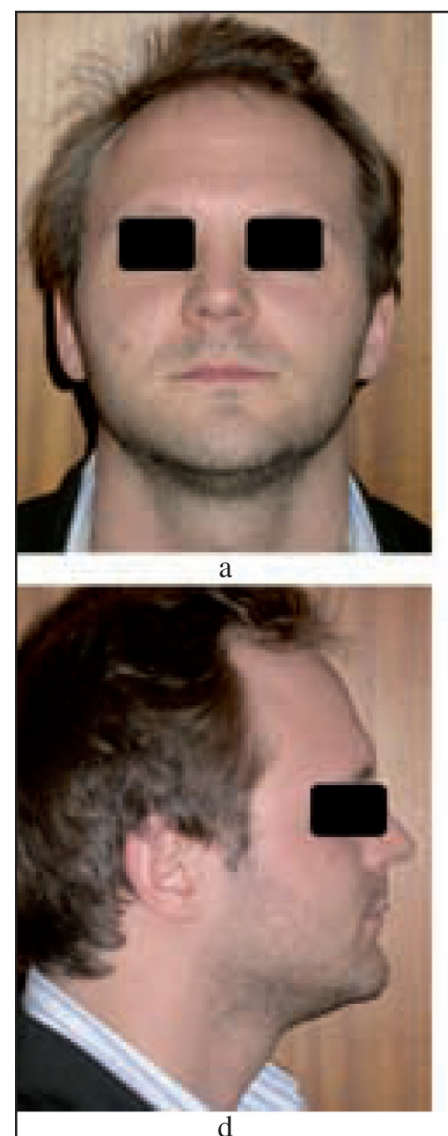

d

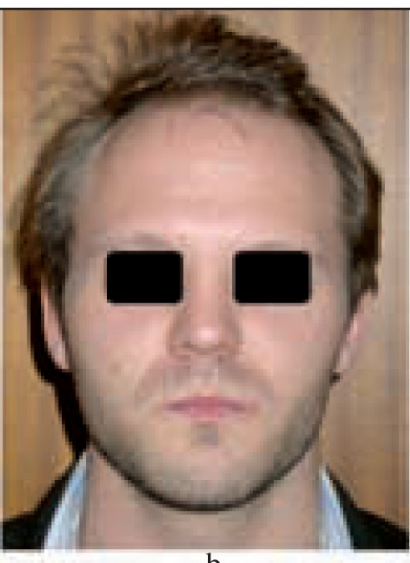

b

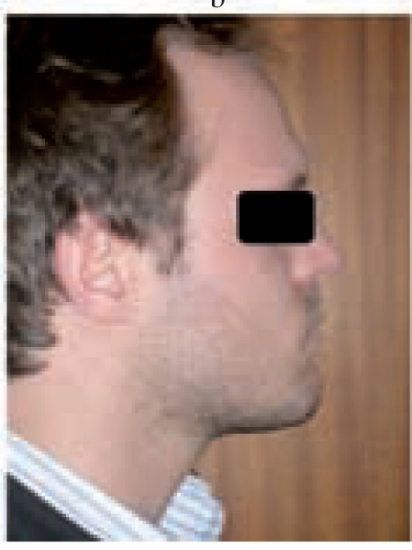

e

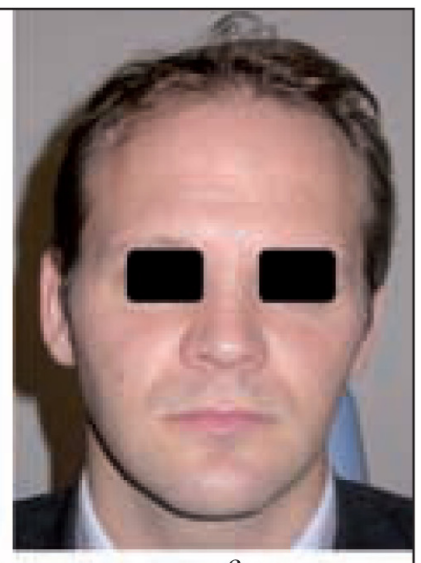

$\mathrm{C}$

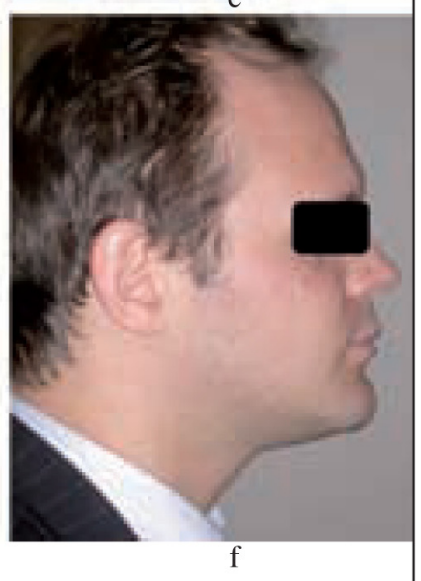

Figures 1 a to $f$

Case of a 30 year old man with severe OSAS (HA/ 30/h), presenting with a dental and ske letal Class II malocclusion. We made pre-treatment esthetic simulation of the maxillo-mandibular advancement by placing a shaped wax insert under the patient's upper lip and asking him to set his mandible in a forward position. The actual result is shown at 3 months post-operatively. 


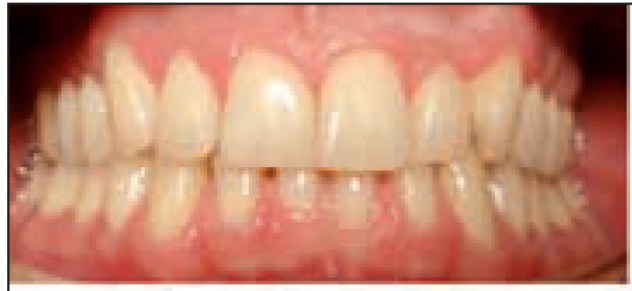

a

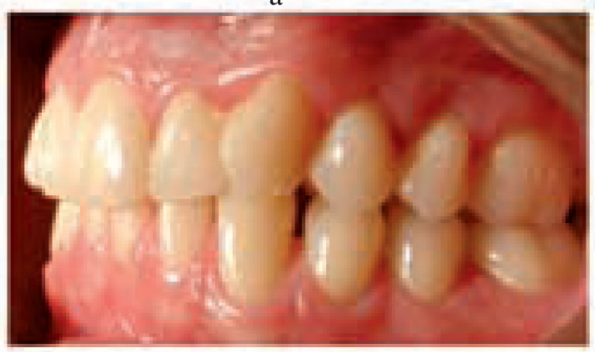

Prt-operatoire

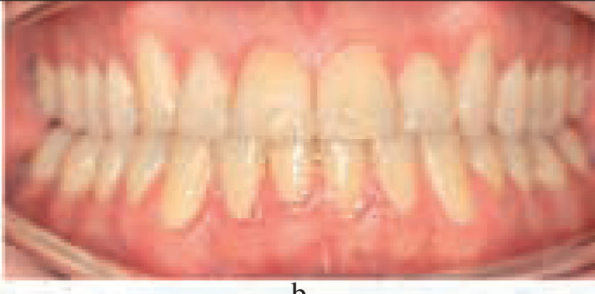

b

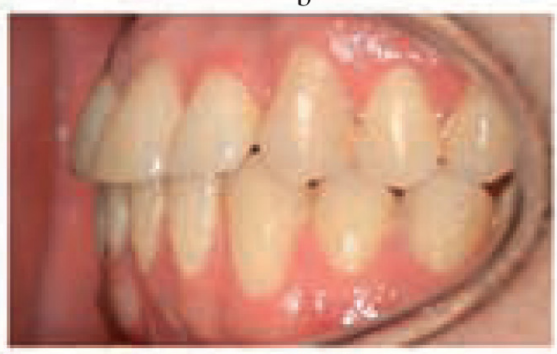

d

Post-optratoire

Figures 2 a to $d$

This patient's Class II Division 2 malocclusion was corrected orthodontically before surgery with buccal torque improving the inclination of the upper anteriors so that the mandible could be advanced sufficiently in surgery and to reduce the extent to which the maxilla had to be moved forward.

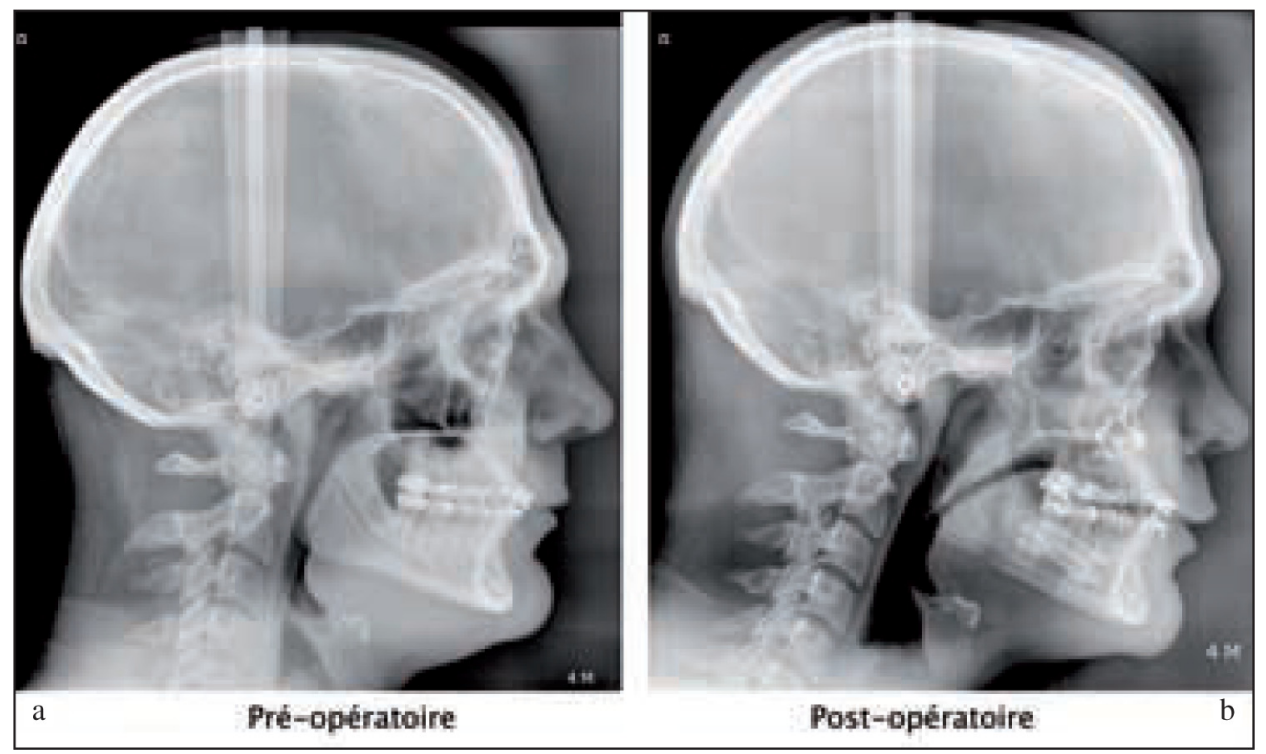

Figures $3 a$ and $b$

A profile cephalogram before surgery. (Note the enlargement of the upper airways and the extent of the skeletal advancement, visible in the area of the osteosynthesis plates). 
Depending upon whether any discrepancies between the two dental arches exist and also on the status of the facial equilibrium, a pre-operative stage of orthodontic treatment may be indicated to improve the final occlusal and esthetic result. In our experience more than half the patients operated on have benefited from orthodontic preparation that fully justified the expectations for it. The protocol that has been established takes into account the constraints inherent in the correction of the apnea and also those of the malocclusions that can be associated with it, the majority of which are of the Class II type.
So the orthodontic correction of these discrepancies permits a greater mandibular than maxillary advancement, which has the effect of minimizing the impact of the maxillary advancement on the naso-labial angle and the columellary projection.

In other cases, maxillary expansion may be necessary because of the inadequate palatal width that characterizes many apnea patients, who are most often mouth breathers (see case 2 , illustrated by figures 4 through 6 ). In most cases it is advisable not to incorporate extractions in the treatment plan in order avoid the risk of reducing the volume of the oral cavity.

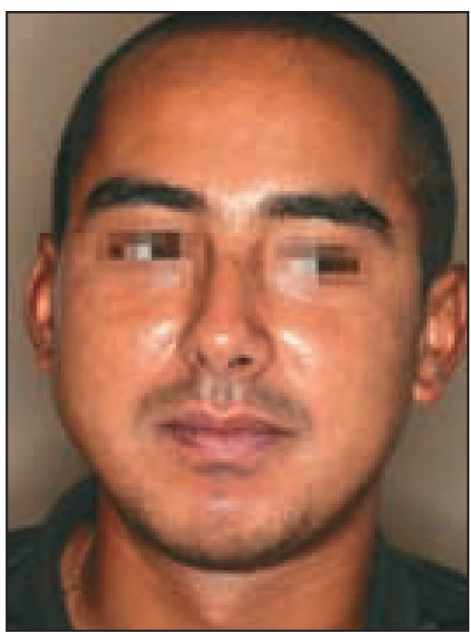

a

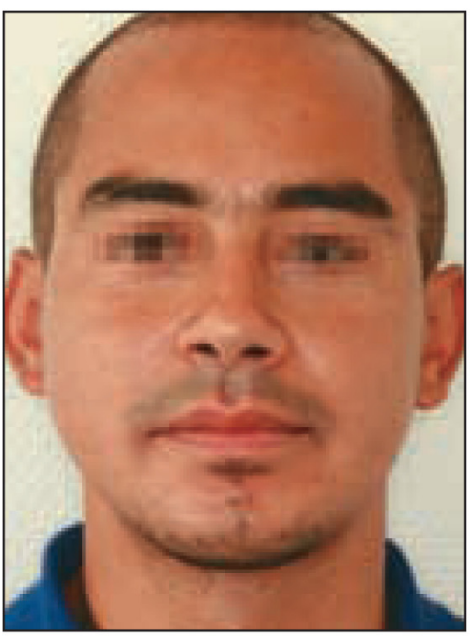

$\mathrm{c}$

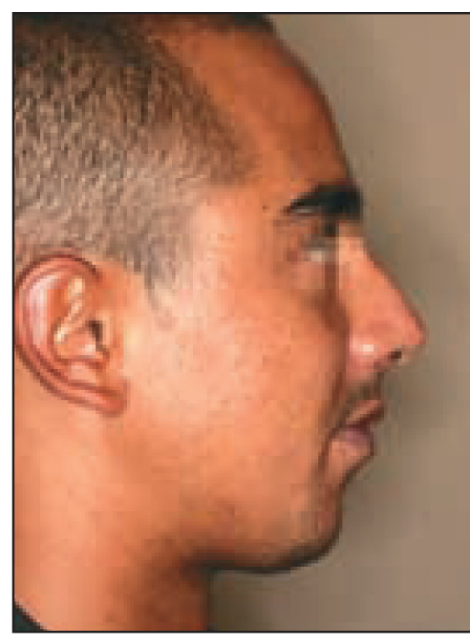

b

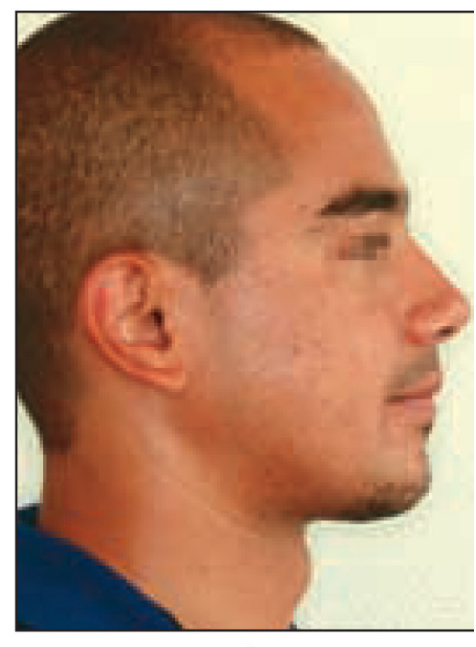

d
Figures 4 a to $d$

This patient with severe OSAS symptoms (IAH 67/h), was treated in a two-stage procedure: surgically assisted rapid palatal expansion followed by maxillo-mandibular advancement surgery. Esthetic changes are shown in full face and profile views before and after the two surgical phases. The orthodontic treatment was completed in 15 months. 


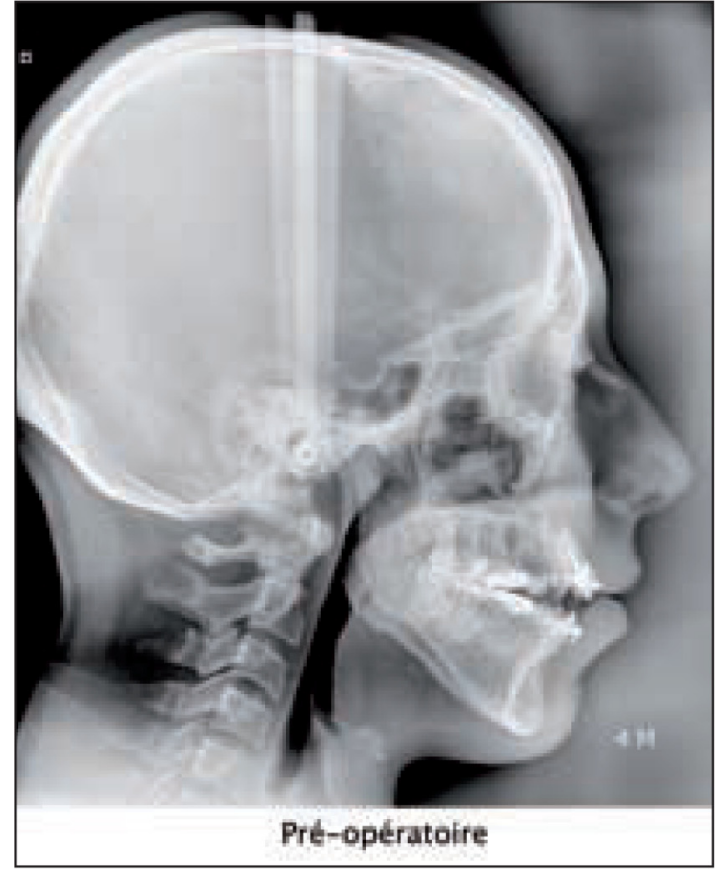

a

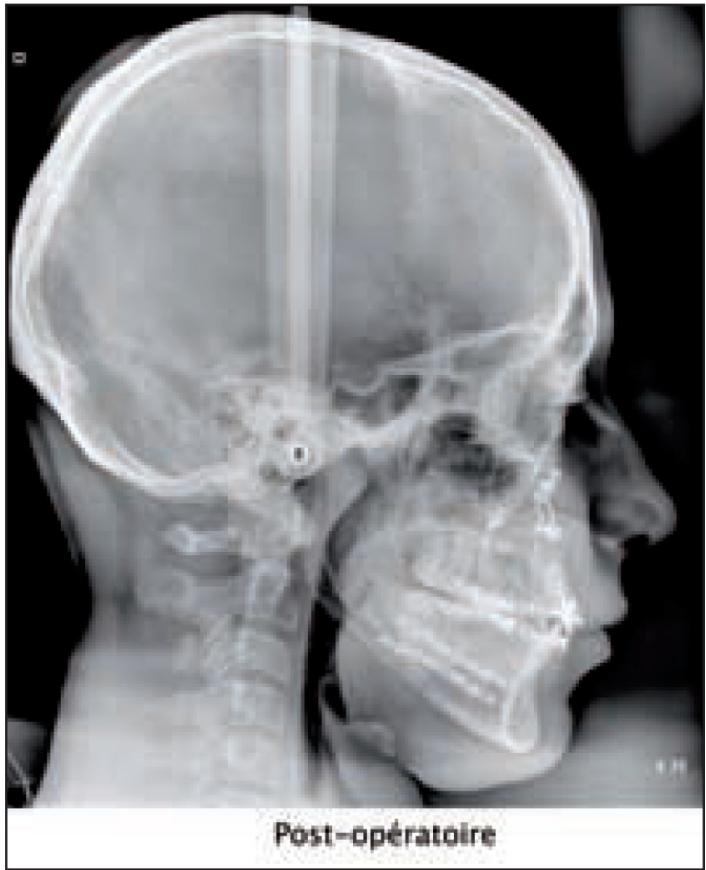

b

Figures 5 a and $b$

Profile cephalograms before (a) and after (b) the second surgical phase that corrected a skeletal open bite, a Class II overjet, and the OSAS symptoms.

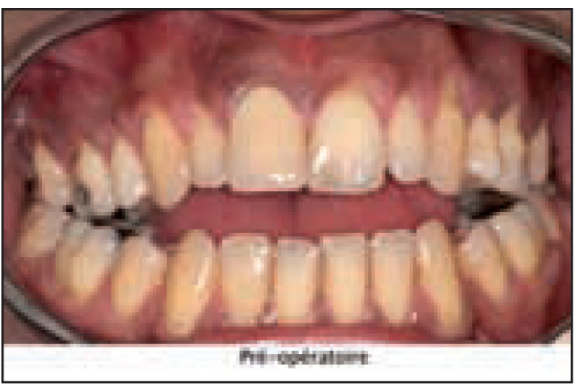

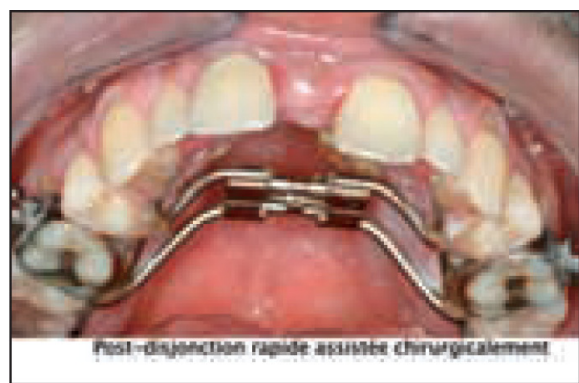

b

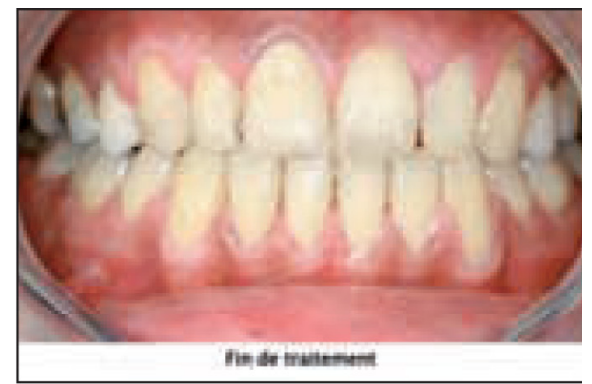

c

Figures 6 a to $c$

a: This patient present initially with a substantial anterior open bite, a maxillary retrusion with bilateral cross bite, and severe gingival recession in the buccal segments. He recalled that in his childhood he had orthodontic treatment with bicuspid extraction, which explained why his teeth were not crowded.

b: The rapid palatal expander, placed after an osteotomy, and activated daily for 8 days post-operatively.

$c$ : The occlusion at the end of treatment. 
These protocols adhere to the guidelines of classic orthodontic treatment, whether buccal or lingual, employing osseous anchorage with miniscrews or plates whenever necessary.

\section{3 - SURGICAL PROTOCOL}

Maxillomandibular advancement osteotomy has the objective of providing a global pharyngeal enlargement by exerting traction on the hyo-lingual muscle complex from its anteriorly propelled insertion in the mandibular

\section{Case 3}
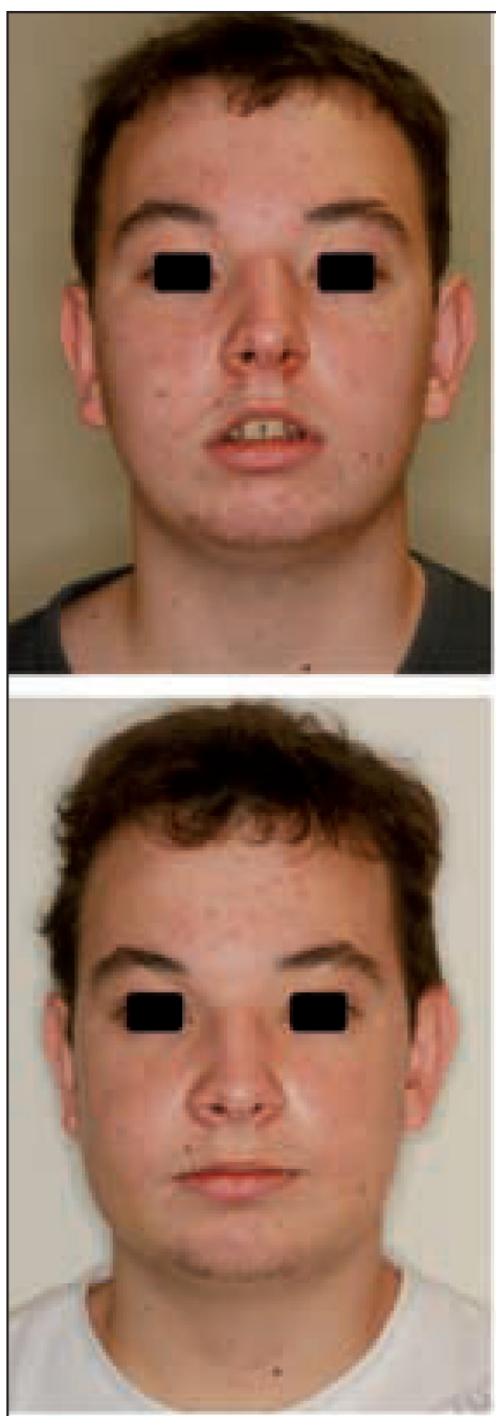

Pré-opetratoire
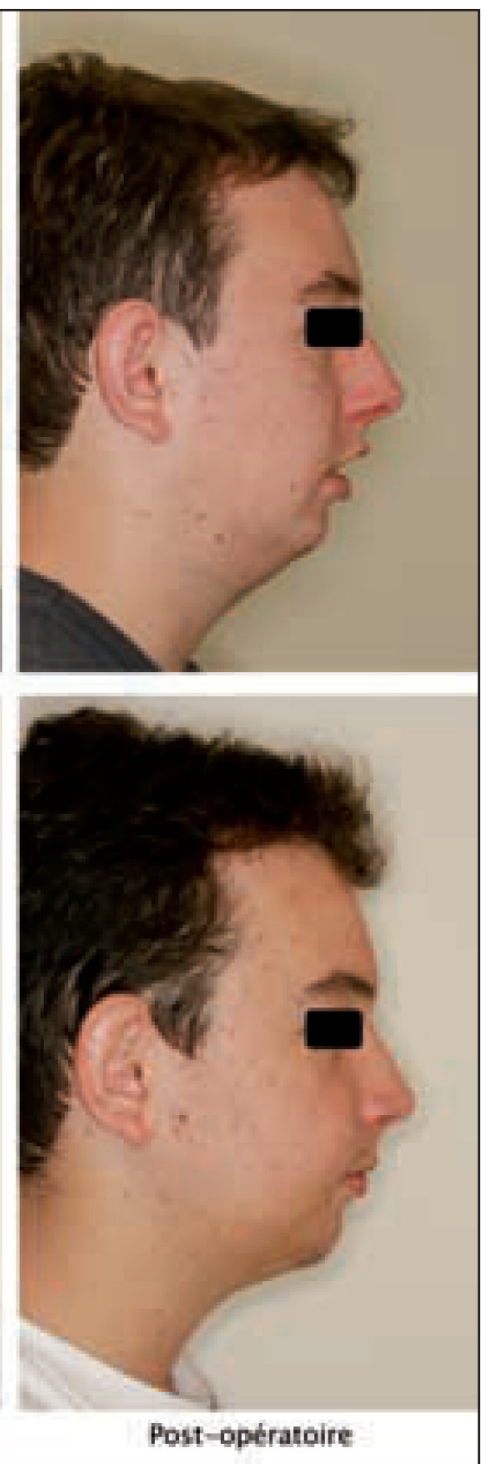

Figure 7

A patient with moderate OSAS, who rejected preliminary full-banded orthodontic treatment. Note the esthetic changes after surgery with the advancement and auto-rotation of maxilla and mandible, which only partially corrected the convexity of his profile. 


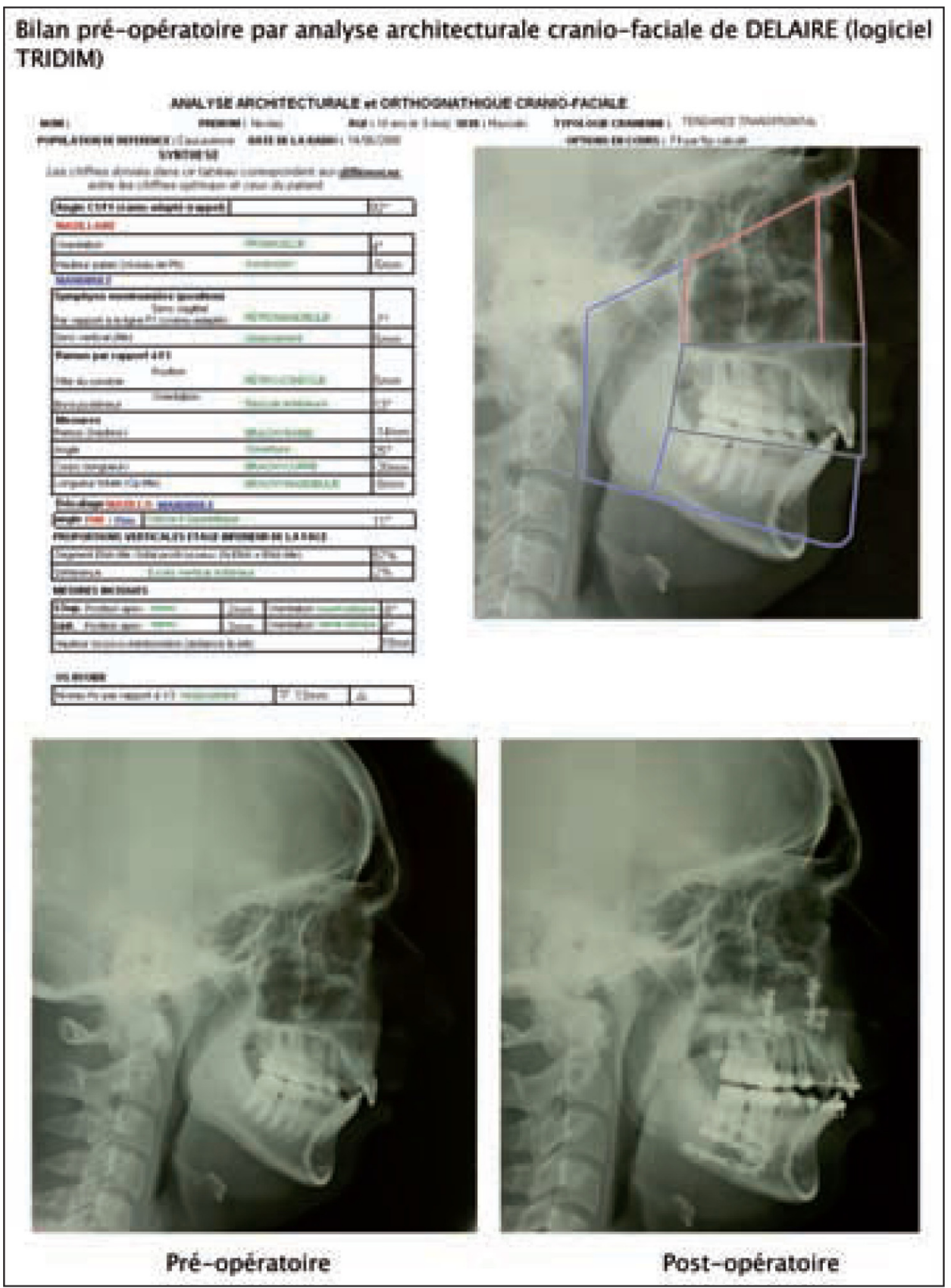

Figure 8

The pre-operative cephalometric assessment carried out by a digitized Delaire (TRIDIM program) cranio-facial architectural analysis. 


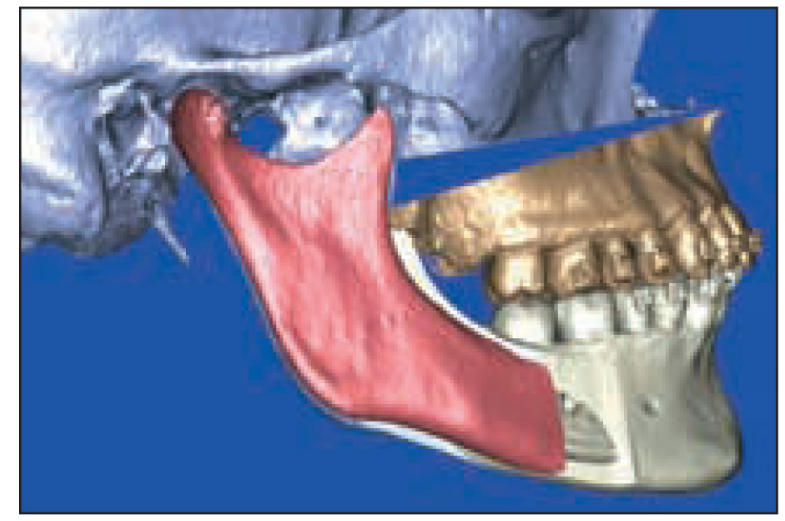

Figure 9

Digitized three dimensional pre-operative simulation made on the basis of an RX scanner from which a numerical surgical guide was constructed.

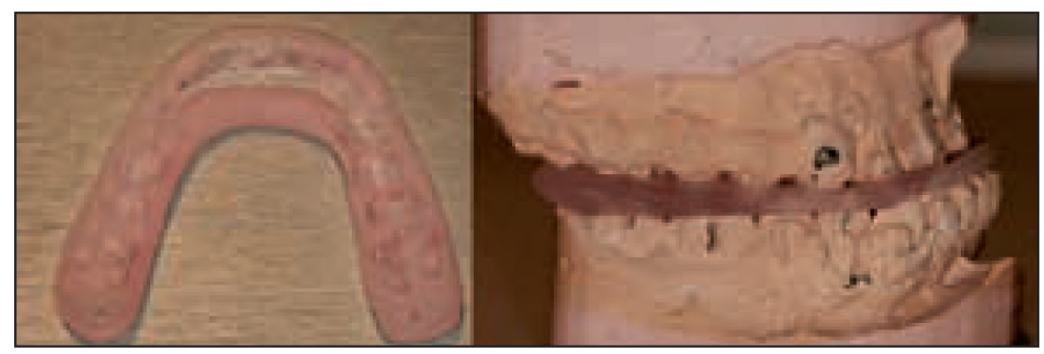

Figure 10

Digitized splint made with information derived from a computer prediction.

symphysis at the genial process and by, at the same time increasing the volume of the oral cavity (see case 3 , figures 7 through 10).

This surgical intervention is derived from the classical Obwegeser Dalpont sagittal mandibular osteotomy, or modified from the Epker type with a Lefort 1 maxillary advancement osteotomy that adjusts the final occlusion to the new position of the lower dental $\operatorname{arch}^{2,8,12,19,20,25,26 . ~}$

To assure osteosynthesis the segments are bound to each other with titanium mini-screws but the jaws are not wired together. Post-operatively patients are followed closely at the same time they receive intensive care adapted to the apnea condition. They can begin eating soft food again 24 hours after the surgery and continue on this diet for two months. In our experience, the average post-operative hospital stay is four days. Full bony union is achieved in about 8 weeks. Patients are checked with a polysomnographic record immediately after surgery and again after 12 months.

No magic formula can be found anywhere in the literature for establishing for each patient the ideal amount of mandibular advancement that would assure the removal of all obstructive respiratory episodes. The extent of advancement in the majority 
of the cases reported is 5 to $10 \mathrm{~mm}$ for the maxilla and 10 to $12 \mathrm{~mm}$ for the mandible $8,9,19,20,22,25,26$.

The difference in magnitude results from the skeletal gap existing in patients who have a pre-existing sagittal malocclusion But no correlation between the extent of the maxillary or the mandibular advancement and a decrease in $\mathrm{HAl}^{22,26}$ has been reported.

\section{4 - INDICATIONS}

Maxillomandibular advancement surgery is indicated as a procedure that can cure patients with severe OSAS symptoms who will not or cannot wear positive air pressure devices or oral device ${ }^{1,21}$.

Traditionally, most writers on the subject have said that maxillomandibular advancement surgery should be limited to OSAS patients with severe symptoms, HAI > 30. However, nowadays when no velo-pharyngeal obstructions are present, it is also being employed for patients with more moderate symptoms who are unable or unwilling to accept more conservative treatment ${ }^{8,9,10,19,20}$.

In selecting patients for this procedure, surgeons should take into account medical and surgical histories, the severity of the apnea condition, the anthropometric and cephalometric data, and an evaluation of the anticipated facial transformation.

It is also recommended that surgeons review a complete cardio-vascular assessment before making any operative decision.
Osteo-distraction is an adjunctive surgical procedure in which bone growth can be achieved in an anteroposterior or transverse dimension by means of a device that allows the surgeon to create a daily increase in the length of the body of the mandible or an expansion of the maxilla. Because it is complex to install and activate and difficult to control its activation, this technique is not currently being widely used ${ }^{3,7,23}$.

Age is an important factor in selection of patients because a decrease in spongy bone' a vital component in osseous remodeling and consolidation is correlated to the age of patients. Operative risk also increases with the age of patients because of the comorbidity rate that is associated with it. The upper limit of the age of patients receiving maxillomandibular advancement surgery reported in the literature is 65 years $2,8.12,1920,25,26$.

Most other authors consider that excess weight causes unsatisfactory results after surgery and suggest that it not be carried out for patients whose obesity level reaches $\mathrm{BMI}>$ $30 \mathrm{~kg} / \mathrm{m}^{29,22}$.

Not all teams working in this field agree that cephalometric criteria are determining factors in decision making $^{8,13}$.

Hochban, in a study based on cephalometric analyses of 403 patients with apnea compared to a control group found an insufficient maxillomandibular projection in $40 \%$ of the patients ${ }^{10}$. He asserted that the 
presence of this anomaly was sufficient reason to undertake maxillomandibular surgery as the primary treatment modality ${ }^{8,9}$ Other treatment teams agree with this position while citing an obstruction site as the determining element ${ }^{19,22,25}$. Li takes an opposing position, affirming that this surgery can be planned for patients with no skeletal deformities in order to obtain results of comparable effectiveness ${ }^{12,15}$.

The Stanford University team proposes a two-stage protocol. In the first step (phase 1): a correction of the oropharyngeal obstruction is obtained by an uvulo-palato-pharyngoplasty (UPPP) associated with a forward placement of the genial process and the hyoid suspension. Should this fail to produce the desired improvement, phase II, which consists of an advancement maxillomaxillary osteotomy can be instituted $12,13,14,20$.

Only $30 \%$ of the patients whose Phase I procedure did not succeed agreed to continue with Phase II, which has led critics of this protocol to allege that placing treatment in a hierarchal order results in a high rate of patients who drop out and are lost from view. They suggest that using cephalometric criteria in the initial selection process would avoid this problem $^{8,19,22,25}$.

\section{5 - RESULTS (tables I and II)}

\begin{tabular}{|c|c|c|c|c|c|}
\hline Authors & Publication & $\mathrm{n}$ & Follow-up months & Criteria for success & Success rate (\%) \\
\hline Waite & $\begin{array}{l}\text { J Oral Maxillofac } \\
\text { Surg } 1989\end{array}$ & 23 & 1,5 & $\mathrm{IAH}<10$ & 65 \\
\hline Riley & $\begin{array}{c}\text { Otolaryngol Head } \\
\text { Neck Surg } 1989\end{array}$ & 55 & 6 & $\mathrm{IAH}<20$ and $50 \% \mathrm{IAH}$ & 67 \\
\hline $\mathrm{Li}$ & Laryngoscope 2000 & 175 & 12 & $\begin{array}{c}\mathrm{IAH}<20 \\
\text { and improvement } \\
\mathrm{IAH} 50 \%\end{array}$ & 95 \\
\hline Bettega & $\begin{array}{c}\text { Am J Respir Crit } \\
\text { Care Med } \\
2000\end{array}$ & 20 & 6 & $\begin{array}{c}I A H<15 \\
\text { less than } 50 \%\end{array}$ & 75 \\
\hline Prinsell & Chest 1999 & 50 & 5,2 & $\begin{array}{c}\mathrm{IAH}<15 \text { and } \mathrm{IA}<5 \\
\text { or } 60 \% \text { reduction } \\
\text { of } \mathrm{IAH} \text { and } \mathrm{IA}\end{array}$ & 100 \\
\hline
\end{tabular}

Table 1

Effectiveness of maxillomandibular advancement osseous surgery (results of studies of groups of more than 20 patients). 


\begin{tabular}{|c|c|c|c|c|c|}
\hline Authors & Publication & Success criteria & Success 6-12 months & Long term follow-up & Long term success \\
\hline $\mathrm{Li}$ & $\begin{array}{c}\text { Sleep Breath } \\
2000\end{array}$ & $\begin{array}{c}\text { IAH }<20 \% \\
\text { and improvement } \\
\text { IAH 50\% }\end{array}$ & $\begin{array}{c}\mathbf{9 5 \%} \\
\mathrm{n}=175\end{array}$ & $\begin{array}{c}50,7 \text { months } \\
\mathrm{n}=40\end{array}$ & $\begin{array}{c}\mathbf{9 0 \%} \\
(36 / 40)\end{array}$ \\
\hline Conradt & $\begin{array}{c}\text { Eur Respir J } \\
1997\end{array}$ & IAH $<10$ & $\begin{array}{c}\mathbf{9 7 \%} \\
\mathrm{n}=38\end{array}$ & $\begin{array}{c}24 \text { months } \\
\mathrm{n}=15\end{array}$ & $\begin{array}{c}\mathbf{8 0} \% \\
(12 / 15)\end{array}$ \\
\hline
\end{tabular}

Table /I

Long term effectiveness of of osseous maxillomandibular advancement surgery.

A number of teams have published the results of this intervention, with effectiveness levels ranging from $65 \%$ to $100 \%$ as measured by a check-up polysomnograph record taken in the first year after surgery.

Lye studied the post-operative changes in the quality of life for a group of 15 patients, 13 of whom had had successful surgical outcomes, after a maxillomandibular advancement procedure with a standardized enquiry "Functional Outcomes of Sleep Questionnaire (FOSQ)" and found significant improvement throughout the gamut of activities, including level of productivity, social behavior, alertness, and libido strength ${ }^{16}$.

A smaller group of investigators have studied the long term stability of the results $5,12,17,18$.

However the two studies that are available reporting on status of patients 2 years and 5 years postoperatively have shown no significant changes in the in the initial results ${ }^{5,12}$.

Only patients who had gained a considerable amount of weight suffered a return of apnea symptoms.

Of the undesirable side effects caused by this surgical procedure, hypo-anesthesia or complete loss of sensation in the lower lip and chin area occur most frequently. This phenomenon is often transitory, but can be the source of sensitive sequellae.

Many investigators have evaluated secondary facial modifications ${ }^{4,6,15}$. None have reported unsatisfactory results that led patients to regret having had this treatment procedure. For patients with retrognathic maxillae, skeletal analyses showed the most favorable esthetic results. Only one study has demonstrated that maxillomandibular advancement surgery did not lead to any major esthetic deficits and that, accordingly, the absence of maxillomandibular retrusion did not constitute a contra-indication for proceeding with this type of surgery ${ }^{15}$.

In our experience, skeletal retrusion, as determined by the Ricketts analysis and concave profiles with an open naso-labial angle are the basic pre-operative components of a favorable facial profile.

On the other hand, a short face, with labial protrusion and a closed naso-labial angle is rather unfavorable. In one study of 15 consecutively treated patients, a jury found that in $70 \%$ of the cases they analyzed the changes were positive ${ }^{24}$. 


\section{6 - CONCLUSION}

Surgeons can achieve, for selected patients, a global and stable correction of the pharyngeal collapse that is responsible for the malady of apnea with maxillomandibular advancement surgery. The procedure is, essentially, contra-indicated for patients over 65 years in age, that suffer from other ailments that would increase the risk excessively, and for whom a simulation of the anticipated postoperative facial change shows that it would not be esthetically satisfactory.

The possibility of predicting the result of surgery by having patients wear advancement oral appliance for a trial period has been described as highly attractive. Such a trial would, in addition, permit patients to use the oral appliance as a substitute for ccontinuous positive airway pressure treatment. The protocols for preparatory orthodontic treatment provide for improving oral function by adjusting the occlusion, limiting the undesirable repercussions of surgical transformation of the face, and, in fact, making that transformation profitable. Taken together, all these elements emphasize the importance of inter-disciplinary collaboration in the effort to treat obstructive sleep apnea.

\section{REFERENCES}

1. American Sleep Disorders Association 1996. Practice parameters for the treatment of obstructive sleep apnea in adults: the efficacy of surgical modifications of the upper airway. Sleep 19(2):152-5.

2. Bettega G, Pepin JL, Veale D, Deschaux C, Raphael B, and Levy P. Obstructive sleep apnea syndrome. fifty-one consecutive patients treated by maxillofacial surgery. Am J Respir Crit Care Med 2000;162(2 Pt 1):641-9.

3. Conley RS and Legan HL. Correction of severe obstructive sleep apnea with bimaxillary transverse distraction osteogenesis and maxillomandibular advancement. Am J Orthod Dentofacial Orthop 2006;129(2):283-92.

4. Conley RS, and Boyd SB. Facial soft tissue changes following maxillomandibular advancement for treatment of obstructive sleep apnea. J Oral Maxillofac Surg 2007;65(7): 1332-40.

5. Conradt R, Hochban W, Brandenburg U, Heitmann J, and Peter JH. Long-term followup after surgical treatment of obstructive sleep apnoea by maxillomandibular advancement. Eur Respir J 1997;10(1):123-8.

6. Goh YH and Lim KA. Modified maxillomandibular advancement for the treatment of obstructive sleep apnea: a preliminary report. Laryngoscope 2003;113(9):1577-82.

7. Guilleminault C, and Li KK. Maxillomandibular expansion for the treatment of sleepdisordered breathing: preliminary result. Laryngoscope 2004;114(5):893-6.

8. Hochban W, Conradt R, Brandenburg U, Heitmann J, and Peter JH. Surgical maxillofacial treatment of obstructive sleep apnea. Plast Reconstr Surg 1997;99(3):619-26; discussion 627-8.

9. Hochban W, Brandenburg $U$ and Peter JH. Surgical treatment of obstructive sleep apnea by maxillomandibular advancement. Sleep 1994;17(7):624-9.

10. Hochban W, and Brandenburg U. Morphology of the viscerocranium in obstructive sleep apnoea syndrome-cephalometric evaluation of 400 patients. J Craniomaxillofac Surg 1994;22(4):205-13. 
11. Hoekema A, de Lange J, Stegenga B and de Bont LG. Oral appliances and maxillomandibular advancement surgery: an alternative treatment protocol for the obstructive sleep apnea-hypopnea syndrome. J Oral Maxillofac Surg 2006;64(6):88691.

12. Li KK, Powell NB, Riley RW, Troell RJ and Guilleminault C. Long-Term Results of Maxillomandibular Advancement Surgery. Sleep Breath 2000;4(3):137-40.

13. Li KK, Riley RW, Powell NB and Guilleminault C. Maxillomandibular advancement for persistent obstructive sleep apnea after phase I surgery in patients without maxillomandibular deficiency. Laryngoscope 2000;110(10 Pt 1):1684-8.

14. Li KK, Riley RW, Powell NB, Troell R and Guilleminault C. Overview of phase II surgery for obstructive sleep apnea syndrome. Ear Nose Throat J 1999;78(11):851, 854-7.

15. Li KK, Riley RW, Powell NB, and Guilleminault C. Patient's perception of the facial appearance after maxillomandibular advancement for obstructive sleep apnea syndrome. J Oral Maxillofac Surg 2001;59(4):377-80; discussion 380-1.

16. Lye KW, Waite PD, Meara D, and Wang D. Quality of life evaluation of maxillomandibular advancement surgery for treatment of obstructive sleep apnea. $J$ Oral Maxillofac Surg 2008;66(5):968-72.

17. Nimkarn Y, Miles PG, and Waite PD. Maxillomandibular advancement surgery in obstructive sleep apnea syndrome patients: long-term surgical stability. J Oral Maxillofac Surg 1995;53(12):1414-8; discussion 1418-9.

18. Pirsig $W$, and Verse $T$. Long-term results in the treatment of obstructive sleep apnea. Eur Arch Otorhinolaryngol 2000;257(10):570-7.

19. Prinsell JR. Maxillomandibular advancement surgery in a site-specific treatment approach for obstructive sleep apnea in 50 consecutive patients. Chest 1999;116(6):1519-29.

20. Riley RW, Powell NB, and Guilleminault C. Maxillofacial surgery and obstructive sleep apnea: a review of 80 patients. Otolaryngol Head Neck Surg 1989;101(3):353-61.

21. Sher AE. The role of maxillomandibular surgery for treating obstructive sleep apnea. Sleep 1996;19(9 Suppl):S88-9.

22. Smatt Yand Ferri J. Retrospective study of 18 patients treated by maxillomandibular advancement with adjunctive procedures for obstructive sleep apnea syndrome. J Craniofac Surg 2005;16(5):770-7.

23. Thompson SH, Quinn M, Helman JI and Baur DA. Maxillomandibular distraction osteogenesis advancement for the treatment of obstructive sleep apnea. J Oral Maxillofac Surg 2007;65(7):1427-9.

24. Vieille E. Esthétique du profil après ostéotomied'avancée maxillo-mandibulaire dans le cadre du syndrome d'apnées obstructives du sommeil. Thèse de Doctorat en Chirurgie Dentaire Paris 7. 2008.

25. Wagner I, Coiffier T, Sequert C, Lachiver X, Fleury B and Chabolle F. [Surgical treatment of severe sleep apnea syndrome by maxillomandibular advancing or mental tranposition]. Ann Otolaryngol Chir Cervicofac 2000;117(3):137-46.

26. Waite PD, Wooten V, Lachner J and Guyette RF. Maxillomandibular advancement surgery in 23 patients with obstructive sleep apnea syndrome. J Oral Maxillofac Surg 1989;47(12):1256-61; discussion 1262. 